\title{
Biogenesis and Function of Circular RNAs in Health and in Disease
}

\author{
George Haddad* and Johan M. Lorenzen* \\ Division of Nephrology, University Hospital Zürich, Zurich, Switzerland
}

\section{OPEN ACCESS}

Edited by:

Frederic Jaisser,

Institut National de la Santé et de la Recherche Médicale (INSERM),

France

Reviewed by:

Andreas Linkermann,

Universitätsklinikum Carl Gustav

Carus, Germany

Max Christoph Liebau,

Klinik und Poliklinik für Kinder- und Jugendmedizin, Universitätsklinikum Köln, Germany

*Correspondence. George Haddad George.Haddad@uzh.ch

Johan M. Lorenzen Johan.Lorenzen@usz.ch

Specialty section:

This article was submitted to

Renal Pharmacology,

a section of the journal

Frontiers in Pharmacology

Received: 29 December 2018

Accepted: 04 April 2019

Published: 26 April 2019

Citation:

Haddad $G$ and Lorenzen JM (2019) Biogenesis and Function of Circular RNAs in Health and in Disease.

Front. Pharmacol. 10:428. doi: 10.3389/fphar.2019.00428
Circular RNAs (circRNAs) are a class of non-coding RNA that were previously thought to be insignificant byproducts of splicing errors. However, recent advances in RNA sequencing confirmed the presence of circRNAs in multiple cell lines and across different species suggesting a functional role of this RNA species. CircRNAs arise from backsplicing events resulting in a circular RNA that is stable, specific and conserved. They can be generated from exons, exon-introns, or introns. CircRNAs have multifaceted functions. They are likely part of the competing endogenous RNA class. They can regulate gene expression by sponging microRNAs, binding proteins or they can be translated into a protein themselves. CircRNAs have been associated with health and disease, some with disease protective effects, some with disease promoting functions. The widespread expression and disease regulatory mechanisms endow circRNAs to be used as functional biomarkers and therapeutic targets for a variety of different disorders. In this concise article we provide an overview of the association of circRNAs with various diseases including cancer, cardiovascular and kidney disease as well as cellular senescence. We conclude with an assessment of the current status and future outlook of this new field of research that carries immense potential with respect to diagnostic and therapeutic approaches of a variety of diseases.

Keywords: circular RNA, non-coding RNA, exosome, platelet, kidney

\section{INTRODUCTION}

Cellular ribonucleic acids are a family of coding and non-coding sequences. Merely $1-2 \%$ of the human genome is transcribed into RNA transcripts, which are translated into protein (Lorenzen and Thum, 2016; Brandenburger et al., 2018). Aside from mRNA which represents 3-7\% (Hastie and Bishop, 1976; Carter et al., 2005) of the total RNA species in mammalian cells, the majority of the RNA species are of the non-coding category that include ribosomal RNA (rRNA) (8090\% Wolf and Schlessinger, 1977; Duncan and Hershey, 1983; Palazzo and Lee, 2015), transfer RNA (tRNA) (10-15\%; Waldron and Lacroute, 1975; Palazzo and Lee, 2015), long non-coding RNA (0.06-0.2\%; Mortazavi et al., 2008; Palazzo and Lee, 2015), microRNA (0.003-0.02\%; Bissels et al., 2009; Palazzo and Lee, 2015), and circular RNA (circRNA) (0.002-0.03\%; Salzman et al., 2012; Palazzo and Lee, 2015) among others. Non-coding RNAs (ncRNAs) are separated into long ncRNAs (lncRNAs, $\geq 200$ nucleotides) and small ncRNAs ( $\leq 200$ nucleotides). Small RNAs including microRNAs, which lead to the repression of gene/protein expression and/or translational inhibition of protein synthesis by post-transcriptional binding of the $3^{\prime}$-untranslated region (UTR) of mRNA targets, have been extensively studied over the past several years (Lorenzen and Thum, 2016; Brandenburger et al., 2018). In contrast, only little information is available regarding 
the functional importance of long non-coding RNAs (lncRNAs). It is becoming evident that lncRNAs are important epigenetic regulators of tissue homeostasis during development and disease (Lorenzen and Thum, 2016; Brandenburger et al., 2018). LncRNAs may be categorized as: (1) sense or (2) antisense, (3) bidirectional promoter (transcribed within $1 \mathrm{~kb}$ of promoters antisense to protein-coding transcript), (4) intronic, (5) intergenic, or (6) enhancer-associated (transcribed from an enhancer region of a protein-coding gene) (Lorenzen and Thum, 2016; Brandenburger et al., 2018). In addition, circular RNAs have been identified as part of the lncRNA class. These RNAs are characterized by a circular structure in which the $3^{\prime}$ and $5^{\prime}$ ends are covalently linked. In the present article we aim to elaborate on the biogenesis of this newly identified RNA class as well as discuss its potential function.

Circular RNAs are diverse RNA species that are found in all live forms from archaea to humans (Danan et al., 2012; Jeck et al., 2013; Haque and Harries, 2017). Although, circRNAs have been discovered over 20 years ago they were initially dismissed for having low abundance or resulting from splicing errors (Salzman et al., 2012; Jeck and Sharpless, 2014; Zheng et al., 2016). However, recent advancements in high throughput sequencing revealed the presence of circRNAs in mammalian cells, across various cell lines and many transcripts are abundant and stable (Jeck and Sharpless, 2014; Haque and Harries, 2017). CircRNAs are generated through a mechanism known as back-splicing "tail" to "head" whereby an exon at the $3^{\prime}$ end of a gene is back-spliced to an exon at the $5^{\prime}$ end of the gene resulting in a circular RNA form (Cocquerelle et al., 1993). CircRNAs are dispersed throughout the genome. They can arise mainly from exons but circRNAs deriving from inter- or intragenic, and intronic regions as well as antisense sequences have been reported (Cocquerelle et al., 1993; Lan et al., 2016).

It is imperative to distinguish valid circular RNA from artifact circular RNA. Jeck and Sharpless (2014) argued that backsplice events can occur not only from exonic circRNAs but also can be generated through other mechanisms such as RTPCR template switching, RNA trans-splicing, as well as tandem duplication. Therefore, methods to validate the circularity of an RNA are essential.

Several methods have been described to separate linear from circRNAs. In general circRNAs do not have a $3^{\prime}$ end as compared to linear exonic RNA and they migrate slower in a two-dimensional polyacrylamide gel (Tabak et al., 1988). RNA degradation by RNase $\mathrm{H}$ or weak hydrolysis can offer a more conclusive method for RNA circularity by linearizing the circRNAs into one product (Capel et al., 1993). In addition, other enzymes that degrade RNA can be employed such as RNase R exonuclease, and terminator exonuclease which have no effect on the circRNAs but degrade the majority of linear RNA (Suzuki et al., 2006; Hansen et al., 2011, 2013; Jeck and Sharpless, 2014).

Similarly, circRNAs must be distinguished from RNA lariats which are generated through canonical RNA splicing and resemble exonic circRNAs. However, lariat RNAs are distinguished by the presence of a $2^{\prime}-5^{\prime}$ junction in contrast to the $3^{\prime}-5^{\prime}$ linkage present in exonic circRNAs. Due to this linkage difference RNA lariats can be depleted by treatment with RNA lariat debranching enzyme followed by exonuclease digestion (Ruskin and Green, 1990; Zhang et al., 2013; Jeck and Sharpless, 2014). CircRNA biogenesis is schematically shown in Figure 1.

\section{Cellular Source of circRNA}

Genome-wide analyses establish a strong evidence of circRNA presence in various cell types (Salzman et al., 2012; Zhang et al., 2013; Jeck and Sharpless, 2014). The expression of circRNAs is prevalent, specific, stable, and displays a temporal and spatial regulation suggesting tissue specific function under physiological and pathological conditions (Wang et al., 2014; Xu et al., 2017; Fang, 2018). They have higher expression levels in low-proliferating cells such as in the brain as compared to high-proliferating cells of the liver (Bachmayr-Heyda et al., 2015). Moreover, in hematopoietic cells including progenitors, differentiated myeloid and lymphoid cells, circRNA expression can be cell specific and increases during cellular maturation. Interestingly, enucleated cells such as red blood cells and platelets appear to express higher levels of circRNAs as compared to nucleated hematopoietic cells (Nicolet et al., 2018). It was found that platelets, in particular, express the highest number of circRNAs, almost twice as much as erythrocytes and 5 times more than granulocytes (Nicolet et al., 2018). Taken together, this suggests that circRNAs may play an important role in maintaining erythrocyte and platelet function in responding to micro-environmental cues and to cross-talk with other cell types through the packaging of circRNAs into microvesicles, or exosomes (Nicolet et al., 2018). Exosomes are vesicles that pinch off the plasma membrane, sized between 40 and $150 \mathrm{~nm}$ in diameter where intracellular materials can be packaged and delivered as messenger shuttles between cells (Properzi et al., 2013; Raposo and Stoorvogel, 2013). Interestingly, the expression levels of select circRNAs in exosomes derived from cancerous tissue is higher than in non-cancer derived exosomes ( $\mathrm{Li} \mathrm{Y}$. et al., 2015). Exosomes are generated through the exosomal pathway that involves the expression of distinct protein markers not present in other vesicles of comparable size. These markers include tetraspanins (TSPAN27, 28, and 29), Tsg101 and Alix from the Rab family of proteins, and heat shock proteins such as HSP70 (Bobrie et al., 2011). The prevalence of circRNAs in extracellular exosomes is proposed to serve as reliable biomarkers in diagnosing various diseases and also assessing the efficacy of a certain treatment (Van Der Pol et al., 2012; Abu and Jamal, 2016).

\section{EXONIC circRNA HYPOTHESIZED FUNCTIONS}

The evidence on the molecular and cellular functions of circRNAs is emerging. However, regarding the vast majority of circular RNA species their biogenesis, targets and regulation are still not well understood (Vicens and Westhof, 2014; Salzman, 2016). The cellular location of certain circRNAs may provide an indication of their function. Many exonic circRNAs with retained introns as well as circular intronic RNAs (ciRNAs) are found predominantly in the nucleus suggesting a transcription regulatory function ( $\mathrm{Li}$ Z. et al., 2015; Huang et al., 2017), 


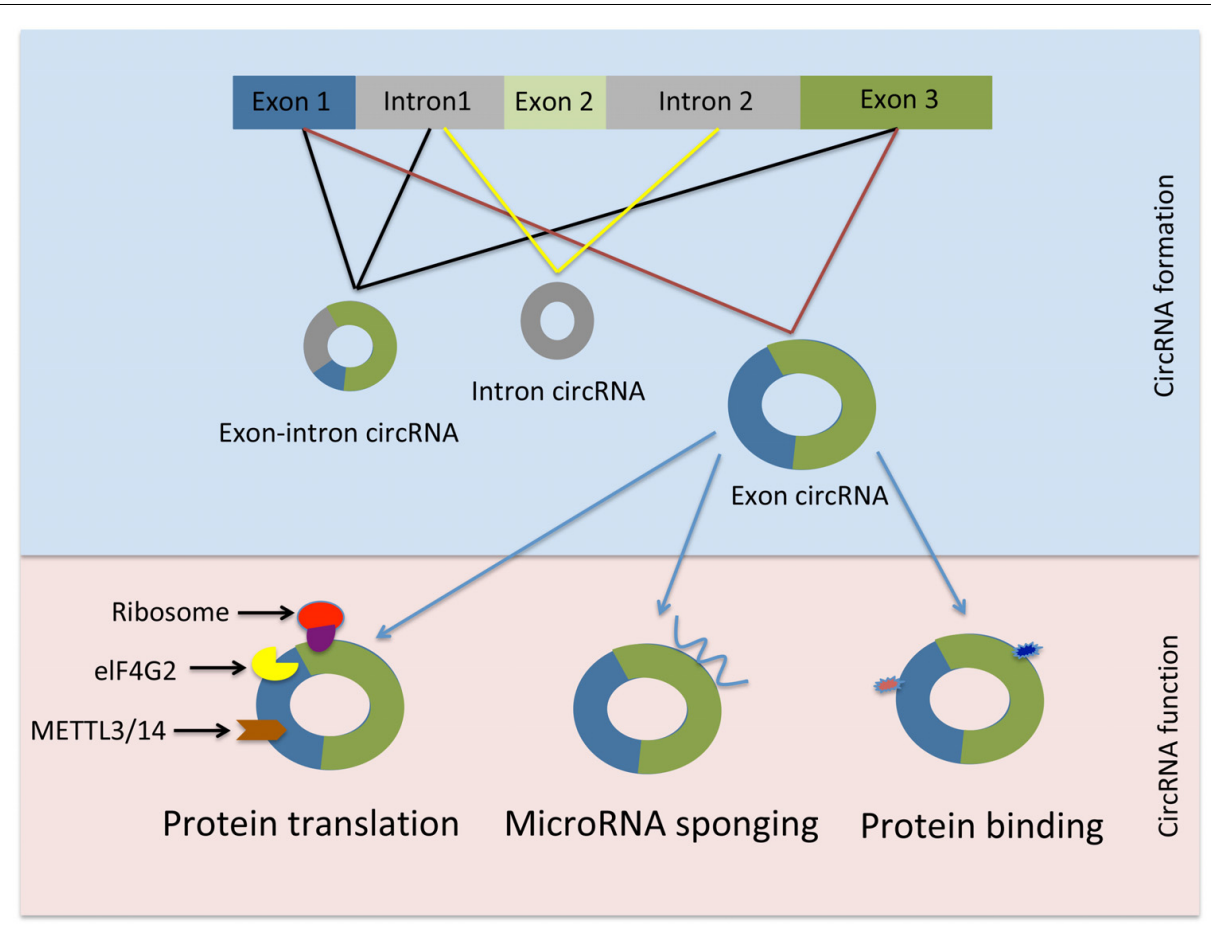

FIGURE 1 | Schematic representation of circRNAs generation and function. elF4G2, initiation factor eukaryotic translation initiation factor 4 gamma; METTL3, methyltransferase-like 3; METTL14, methyltransferase-like 14.

whereas, circRNAs that are generated from exons only are mostly found in the cytoplasm, which may insinuate a role in posttranscriptional gene regulation (Salzman et al., 2012; Jeck et al., 2013; Wilusz, 2018).

The circRNA-sponging function of miRNA is well characterized in the literature (more examples are provided in this review in the following sections). However, it should be noted that merely binding of circRNA to a miRNA does not always result in miRNA suppression. For example, when ciRS-7 is bound to miR-671 it becomes susceptible to cleavage by Argonaute 2 (AGO2), thus releasing miR-7 in the process. Therefore, circRNAs may act as a miRNA reservoir or mediate their transportation (Kristensen et al., 2018).

Interestingly, certain circRNAs have the potential to be translated into protein in a process that requires $\mathrm{N}^{6}$ methyladenosine $\left(\mathrm{m}^{6} \mathrm{~A}\right)$ RNA base modification, the initiation factor eukaryotic translation initiation factor 4 gamma (elF4G2), methyltransferase-like 3 (METTL3), and methyltransferaselike 14 (METTL14). Mass spectrometry and computational prediction analyses demonstrated that there are hundreds of endogenous circRNAs with the potential to be translated into protein (Yang et al., 2017). For instance, circ-FBXW7 encodes a $21 \mathrm{kDa}$ protein known as F-Box and WD Repeat Domain Containing 7 (FBXW7) - 185aa. Overexpression of this novel protein reduced cellular proliferation and repressed glioma tumorigenesis (Yang Y. et al., 2018).

Moreover, circRNA function may be extended to include protein-binding activity and it has been suggested that circRNAs are likely to be involved in protein binding, sorting, sequestering, and modulating protein-protein interactions through scaffolding properties. Based on bioinformatic analyses many circRNAs are predicted to harbor RNA binding protein sites (Du et al., 2017b). However, due to the tertiary structure of circRNAs the protein binding function appears to be a more complicated process than previously thought (Du et al., 2017b). Nevertheless, there are few reports demonstrating the protein binding capacity of circRNAs. For instance, Argonaute proteins, which are involved in miRNA trafficking, were found to strongly interact with human $\operatorname{circRNA}$ cerebellar degeneration-related protein 1 transcript (CDR1as) by using the technique photoactivatable-ribonucleoside-enhanced crosslinking and immunoprecipitation or PAR-CLIP (Memczak et al., 2013). Additionally, the circRNA circMbl generated from exon 2 of the splicing factor muscleblind (MBL/MBNL1) in both drosophila and humans carries highly conserved binding sites for the RNA binding protein MBL in both the flanking introns and within the circularized exon 2. The authors further demonstrated a strong and specific binding of $\mathrm{MBL}$ to the circRNA, and this interaction was essential for increased circMbl expression by exogenous expression of MBL (Ashwal-Fluss et al., 2014). CircRNAs as well as their various functions are summarized in Table 1.

\section{circRNAs as Regulator of miRNA Function}

There is a breadth of evidence to support the sponging effect (as competing endogenous RNA) regarding miRNAs of circRNAs in a tissue or organ specific manner. The following section 
TABLE 1 | Summary of circRNAs and their functions in health and disease.

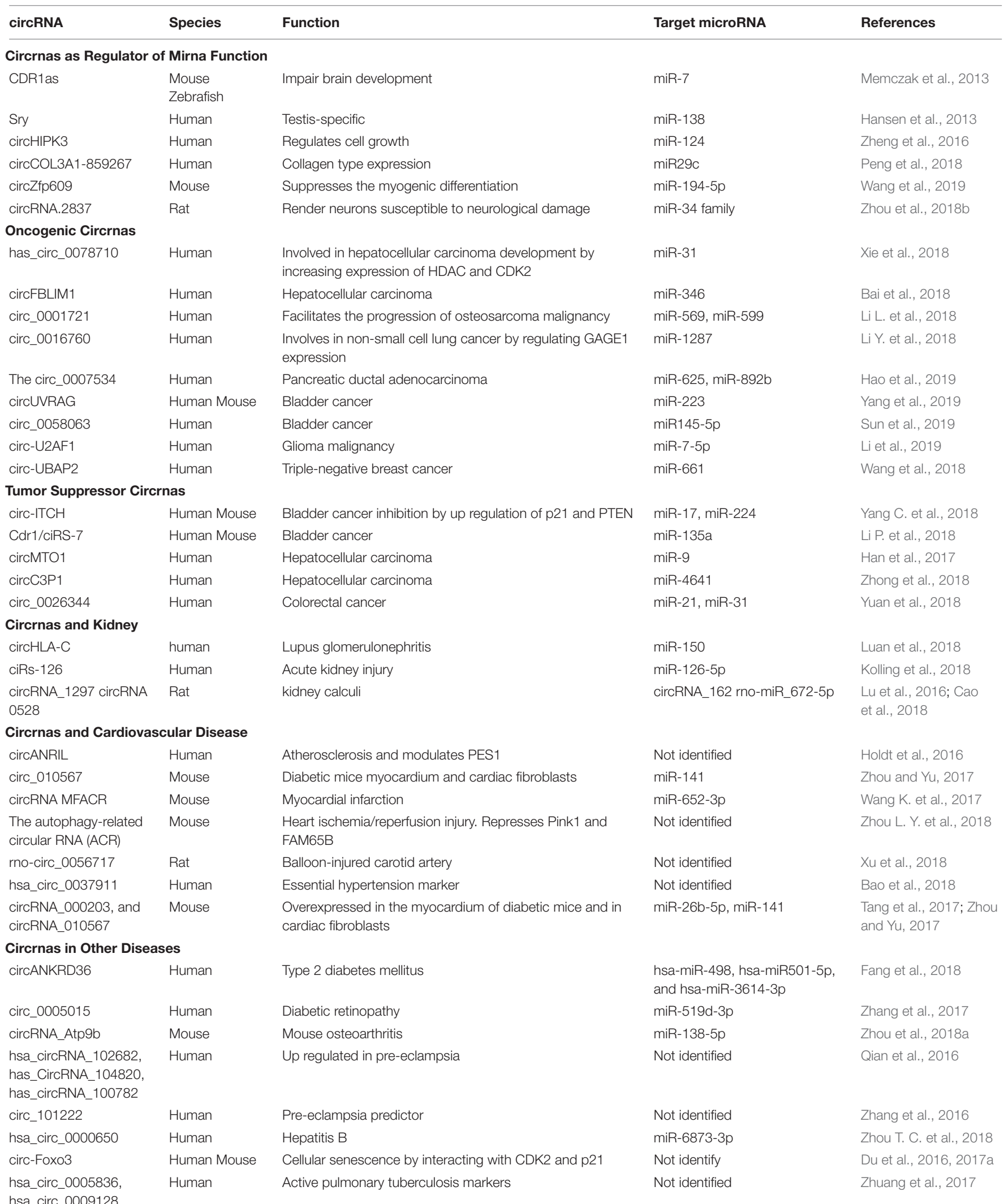


describes several selected examples that demonstrate the specific sponging role of circRNAs in various tissues. The circRNA sponging function was elegantly demonstrated by Memczak et al. in their seminal study. They described the sponging function of a circRNA CDRlas or ciRS-7. This circRNA was shown to harbor 74 miR-7 seed matches suggesting a strong interaction between this circRNA and its miRNA target. The interplay between CDR1as and miR-7 was demonstrated to be specific to neuronal tissue in adult and embryonic mouse brain. CDR1as overexpression and the resulting inhibtion of miR-7 by morpholinos in zebrafish resulted in reduction of midbrain size (Memczak et al., 2013). Similarly, the testis specific circRNA sexdetermining region $Y$ (Sry) was shown to particularly sponge miR-138 and regulate its function in an in vitro study using HEK293 cellls (Hansen et al., 2013). The circRNA derived from exon 2 of the homeodomain-interacting protein kinase 3 (HIPK3) gene, circHIPK3 is thought to have the potential to sponge up to 9 different miRNAs with an estimation of 18 binding sites. There was a significant inhibition of cell growth upon circHIPK3 silencing in the human hepatocyte cell line $\mathrm{HuH}-7$. Moreover the authors demonstrated direct binding to and sponging of miR-124 thus regulating its activity (Zheng et al., 2016). The expression of type 1 collagen is regulated by circCOL3A1-859267 in human dermal fibroblasts. Although this circRNA contains putative binding sites to several miRNA (e.g., miR29a-c, miR767, and miR-133a), only miR29c was identified to be a target of circCOL3A1-859267. Overexpression of miR29c decreased type 1 collagen expression. However, this effect was abrogated by the introduction of circCOL3A1-859267 using a lentivirus expression system (Peng et al., 2018). In skeletal muscle development the circular RNA zinc finger protein 609 (circZfp609) suppressed the myogenic differentiation in mouse myoblast cell line $\mathrm{C} 2 \mathrm{C} 12$ by sponging up miR-194-5p that regulates the expression of BCL2associated transcription factor (BCLAF1) (Wang et al., 2019). Lastly, the suppression of circRNA.2837 expression may protect neurons from neurological damage in a rat sciatic nerve injury model since circRNA.2837 acts as a sponge to members of the miR-34 family (Zhou et al., 2018b).

\section{circRNAs and Cancer}

Since the identification of circRNA sponging of miRNA that are involved in cancer pathogenesis, a multitude of studies have been forwarded regarding the association of circRNAs with various human cancers (Kristensen et al., 2018). Given the biomarker potential of circRNAs as well as the their proposed future use as therapeutic agents, it is imperative to improve our understanding how circRNAs and their miRNA targets are regulated in the development and progression of cancer. Therefore, in this review we will highlight the current findings that implicate certain circRNAs in cancer development and growth, in contrast to other circRNAs that act as tumor suppressors.

\section{Oncogenic circRNAs}

Several studies have outlined the sponging activity of certain circRNAs with regard to miRNAs, which are critical regulators of oncogenes in tumor development. For instance, overexpression of hsa_circ_0078710 increased cellular proliferation and migration, tumor growth and migration in human hepatocellular carcinoma (HCC) cell lines HepG2 and SMMC-7721 by sponging miR-31, increasing the expression levels of histone deacetylase 2 (HDAC2) and cyclin-dependent kinase 2 (CDK2) (Xie et al., 2018). In another HCC study the downregulation of the circRNA filamin-binding LIM protein 1 (circFBLIM1) reduced cellular proliferation and invasion, and concomitantly induced apoptosis in human HCC cell lines HepG2, 7402, and $97 \mathrm{H}$. CircFBLIM1 acts as a competitive endogenous RNA to filamin-binding LIM protein 1 (FBLIM1) and by binding to miR-346 which targets FBLIM1 mRNA (Bai et al., 2018). Circ_0001721 sponges miR-569 and miR-599 and facilitates the progression of osteosarcoma malignancy in humans. Therefore, the expression of circ_0001721 can be used as an unfavorable predictor of disease progression in osteosarcoma patients ( $\mathrm{Li} \mathrm{L}$. et al., 2018). The oncogenic effect of circ_0016760 is attributed to the sponging activity of miR-1287 and the increased expression of cancer/testis antigen family 4 member 1 (GAGE1) in nonsmall cell lung cancer human cell lines A549 and H1299 (Li Y. et al., 2018). Circ_0007534 is found upregulated in patients with pancreatic ductal adenocarcinoma (PDAC). Overexpression of this circRNA was associated with advanced tumor stage and invasion of the lymph node. Further analysis revealed that overexpression of circ_0007534 in the human PDAC cell line SW1990 led to increased migration, proliferation, and invasion. Circ_0007534 mediates its oncogenic effect by sponging miR625 , and miR-892b (Hao et al., 2019). The downregulation of the circRNA UV radiation resistance-associated gene (circUVRAG) expression suppressed proliferation and metastasis of bladder cancer in vitro using the human bladder cancer cell line UM-UC3 and in vivo using Balb/c nude mice injected with lentivirally stabilized circUVRAG UM-UC-3 into the right flank. The effects of circUVRAG are attributed to a mechanism that involves miR-223 sponging and downregulation of fibroblast growth factor receptor 2 (FGFR2) (Yang et al., 2019). Similarly, the oncogenic effect of circ_0058063 was demonstrated in another bladder cancer study. The suppression of circ_0058063 reduced cellular proliferation and migration and induced apoptosis in the human bladder cancer cell lines T24 and J82. The sponging of miR145-5p, which regulates cyclin-dependent kinase (CDK6), is a key element of the function of circ_0058063 in the development and progression of bladder cancer (Sun et al., 2019). In a human glioma malignancy study the circRNA U2 auxiliary factor $35 \mathrm{kDa}$ subunit (circ-U2AF1) was found upregulated along with neuro-oncological ventral antigen 2 (NOVA2). Suppression of circ-U2AF1 resulted in decreased expression of NOVA2 and an increase in expression of miR-7-5p both in vitro and in vivo. As a result, silencing of circ-U2AF1 decreased cellular proliferation, migration and invasion, and increased cell death by apoptosis in the human glioma cell lines U87MG, and U251. Luciferase assay demonstrated that miR-7-5p is a direct target of circ-U2AF1 (Li et al., 2019). In triple-negative breast cancer (TNBC) circ-UBAP2 was found upregulated and associated with larger tumor size. The microRNA miR-661, which regulates the expression of the metastasis-associated gene (MTA1), was found to be a direct target of circ-UBAP2. Suppression of circ-UBAP2 induced apoptosis and decreased proliferation and migration 
of the human TNBC cell lines BT-20 and MDA-MB-231 (Wang et al., 2018).

\section{Tumor Suppressor circRNAs}

Many studies have shown the remarkable potential that circRNAs may have in the future as an anti-cancer treatment option. The anti-tumor activity of the circRNA itchy E3 ubiquitin protein ligase (circ-ITCH) was demonstrated in human bladder cancer tissue and in human bladder cancer cell lines EJ and T24. The overexpression of circ-ITCH inhibited cellular migration, proliferation, and metastasis in vitro and also suppressed cancer formation of xenografts in Balb/c nude mice in vivo. The mechanism of circ-ITCH function is demonstrated by the upregulation of the tumor suppressor genes p21 and phosphatase and tensin homolog (PTEN) expression due to the sponging effect of circ-ITCH to both miR-17 and miR-224 (Yang C. et al., 2018). Similarly, in another bladder cancer study the circRNA Cdr1 (also known as ciRS-7) is downregulated in cancer cells as compared to normal adjacent tissue. Overexpression of Cdr1 reduced cellular proliferation, migration, and invasion in the human bladder cancer cell lines EJ and T24 in vitro, and retarded tumor growth in Balb/c nude mice in vivo. The observed anti-oncogenic effect of Cdr1 is attributed to the sponging function of miR-135a (Li P. et al., 2018). Han et al. performed a global circRNA expression analysis using 289 human samples of HCC and paired adjacent liver tissues to identify dysregulated circRNA expression in HCC samples. Here, the circRNA mitochondrial tRNA translation optimization 1 (circMTO1) was found to be significantly downregulated. Further analysis showed that suppression of circMTO1 led to increased proliferation and invasion of the human HCC cell lines HepG2 and SMMC-7721. CircMTO1 exerts its anti-tumor activity by sponging oncogenic miR-9 and thus preserving the expression of p21 (Han et al., 2017). The expression level of the circRNA complement component 3 precursor pseudogene ( $\operatorname{circC} 3 \mathrm{P} 1$ ) is significantly downregulated in HCC and the expression level of this circRNA is inversely correlated with tumor size and vascular invasion. In contrast, overexpression of circC3P1 decreased cellular migration, proliferation and invasion in human HCC Hep3B and MHCC97-L via a mechanism that involves sponging of miR-4641 and increased expression of phosphoenolpyruvate carboxykinase 1 (PCK1) (Zhong et al., 2018). Yuan et al. screened 32 pairs of human colorectal cancer (CRC) tissues and adjancent normal tissues and identified circ_0026344 to be downregulated in CRC tissues, whereas miR-21 and miR-31 expression levels were elevated. The ectopic expression of circ_0026344 induced apoptosis and reduced tumor growth and invasion in human CRC cell lines SW480 and HT29 via a mechanism that involves miR-21 and miR-31 sponging (Yuan et al., 2018).

\section{circRNAs and Kidney}

The function of circRNAs in kidney homeostasis and disease pathogenesis remains largely unexplored. The few available studies, however, suggest that circRNAs may be used as promising biomarkers in blood (Memczak et al., 2015) and urine (Vo et al., 2019) of patients and may serve as therapeutic targets in kidney disease. Luan et al. performed a circRNA profiling in 6 kidney biopsies of patients with lupus nephritis (LN) compared to 5 healthy kidney tissues. Consequently, 171 circRNAs with at least twofold differential expression were identified. Interestingly, the expression of circHLA-C correlated positively with renal activity index, serum creatinine, proteinuria, and crescentic glomureli. The expression of circHLA-C in patients with LN was increased 2.72-fold compared to controls. This increased expression coincided with a $66 \%$ reduction in expression of miR150 in LN patients. The authors argued that the sponging of miR-150 could be a factor in the pathogenesis of LN (Luan et al., 2018). In a global circRNA analysis of RNA extracted from blood of patients with acute kidney injury (AKI) three novel circRNAs were upregulated in the blood of AKI patients as compared to control. Most notably, the circRNA circular RNA sponge of miR-126 (ciRs-126) was found to be an independent predictor of 28 days survival of patients with acute kidney injury (AKI). In this study miR-126-5p was downregulated in AKI patients and in hypoxic endothelial cells. This effect is attributed to the potential sponging function of ciRs-126 of its target miR-126-5p (Kolling et al., 2018). Wang et al studied the expression of circRNAs in uremia due to glomerulonephritis. They screened a total of 20 individuals, 10 healthy controls as well as 10 patients without systemic lupus erythematosus, for the presence of circRNAs in plasma and peripheral mononuclear cells (PBMCs). The research group identified 385-upregulated and 325 downregulated circRNAs in plasma. In addition, 670 upregulated and 298 downregulated circRNAs were found in PBMCs. The differentially expressed circRNAs relate to various cellular functions including signal transduction, migration, cell differentiation, immune responses and other functions. For example, the regulated circRNAs target genes include ring finger protein 41 (RNF41), ST6 beta-galactoside alpha-2,6sialtransferase (ST6GAL1), WD repeat-containing protein 37 (WDR37), notch homolog 1, translocation-associated (Notch), protein phosphatase 2, regulatory subunit $\mathrm{B}^{\prime}$, alpha subunit (PPP2R5A), adaptor related protein complex 4 subunit $\mathrm{Mu} 1$ (AP4M1), and presenilin 1 (PSEN1). The authors suggest that this study could potentially provide diagnostic biomarkers for the diagnosis and prognosis of patients with uremia (Wang X. et al., 2017). The expression profiles of circRNAs in normal and fetal human tissues using RNA-seq identified 474 circRNAs with adult kidney specific expression and 7962 circRNAs expressed in the human fetal kidney. These results suggest that circRNAs function in a tissue specific manner (Xu et al., 2017). In a rat model of ethylene glycol-induced kidney calculi the expression profiles of mRNA, circRNAs linear IncRNAs were determined. In this animal model 145 circRNAs were deregulated in rat kidney with urolithiasis. Interestingly, circRNA_1297 was associated with rno-miR-138-5p, but circRNA_0528, and circRNA_162 were associated with rno-miR_672-5p. The two microRNAs have been demonstrated to play a role in the development of hypercalciuria urolithiasis (Lu et al., 2016; Cao et al., 2018).

\section{circRNAs and Cardiovascular Diseases}

The role of circRNA in the pathogenesis of cardiovascular diseases is a heavily reviewed topic (Fan et al., 2017; Althesha et al., 2018; Carrara et al., 2018; Holdt et al., 2018a,b; Stępień 
et al., 2018; Zhou M. Y. et al., 2018). Therefore a summary of the major findings will be described here. The atherosclerosis protective circRNA antisense non-coding RNA in the INK4 locus (circANRIL) modulates the function of pescadillo homologue 1 (PES1), an important 60S-preribosomal assembly factor, and protects against atherogenesis by modulating ribosomal RNA (rRNA) maturation in human smooth muscle cells and macrophages, thereby inducing apoptosis and inhibiting cellular proliferation (Holdt et al., 2016). In a mouse study, the expression of circ_010567 was increased in diabetic mice myocardium and cardiac fibroblasts via a mechanism that involved miR141 sponging and upregulation of transforming growth factor beta 1 (TGF $\beta 1$ ) expression. The suppression of circ_010567 expression reduced fibrotic protein expression such as Collagen 1, Collagen 3 and alpha smooth muscle actin ( $\alpha \mathrm{SMA})$ in mouse cardiac fibroblasts, (Zhou and Yu, 2017). The circRNA MFACR (mitochondrial fission and apoptosis-related circular $R N A$ ) targets miR-652-3p specifically that leads to mitochondrial fission inhibition and a reduction of cardiomyocyte apoptosis by decreasing the expression of mitochondrial protein 18 (MTP18). In a mouse model of myocardial infarction (MI), suppression of MFACR in cardiomyocytes decreased mitochondrial fission and notably myocardial infarction (Wang K. et al., 2017). The function of circRNAs in the regulation of autophagy in cardiovascular diseases was demonstrated by Zhou et al. The Autophagy-related circular RNA (ACR) has a protective role in the heart against ischemia/reperfusion injury in a mouse model of myocardial infarction by repressing autophagy through a process that involves PTEN induced putative kinase 1 (Pink1) activation, family with sequence similarity 65 , member $B$ (FAM65B) phosphorylation, and the blocking of Pink1 promoter DNA methylation mediated by DNA methyltransferase 3 beta (Dnmt3B) (Zhou L. Y. et al., 2018). In a rat model of ballooninjury of the carotid artery, the diaphanous related formin 3 (Diaph3) derived circRNA rno-circ_0056717 (circDiaph3) was identified in a microarray and the suppression of this circRNA increased insulin-like growth factor 1 receptor (Igf1r) expression and stimulated vascular smooth muscle cell proliferation and migration (Xu et al., 2018). In an essential hypertension (EH) study that included 200 patients, the circRNA hsa_circ_0037911 was significantly overexpressed in $\mathrm{EH}$ patients and the authors suggest that this circRNA could be an effective marker of EH (Bao et al., 2018). The circRNAs circRNA_000203, and circRNA_010567 are overexpressed in the myocardium of diabetic mice and in cardiac fibroblasts in a cardiac fibrosis mouse model related to Angiotensin II (Ang II). Overexpression of circRNA_000203 in mouse cardiac fibroblasts resulted in a promotion of the expression of collagen-1- $\alpha 2$ (colla2), collagen$3-\alpha 1$ (col3a1) and $\alpha$ SMA via a mechanism of sponging miR26b-5p. Additionally, the downregulation of circRNA_010567 in cardiac fibroblasts resulted in suppression of TGFb1 expression, which is a target of miR-141, and the decreased expression of fibrosis related genes (Tang et al., 2017; Zhou and Yu, 2017).

\section{circRNAs in Other Diseases}

The role that circRNAs play in disease protection or development extends to several other diseases that have been already reported.
For instance, the circRNA derived from the gene ankyrin repeat domain-containing protein 36A (circANKRD36) was overexpressed in peripheral leucocytes of patients with type 2 diabetes mellitus (T2DM). The expression of circANKRD36 positively correlated with higher glucose and glycosylated hemoglobin levels. In addition, the expression levels of interleukin-6 (IL-6) and tumor necrosis factor alpha (TNFalpha) were significantly increased in the peripheral blood of patients with T2DM. The authors suggest an involvement of circANKRD36 in the increased inflammatory response in T2DM patients through a mechanism that involves the regulation of miRNAs, hsa-miR-498, hsa-miR501-5p, and hsa-miR-3614$3 p$ (Fang et al., 2018). In diabetic retinopathy patients the expression of circ_0005015 was elevated in the fibrovascular membranes, the plasma and the vitreous sample, which resulted in enhanced endothelial angiogenic responses via sponging miR$519 \mathrm{~d}-3 \mathrm{p}$ and increased expression of matrix metallopeptidase 2 (MMP-2), signal transducer and activator of transcription 3 (STAT3), and X-linked inhibitor of apoptosis (XIAP) (Zhang et al., 2017). The function of circRNA derived from the gene ATPase phospholipid transporting 9B (circRNA_Atp9b) was examined in a mouse osteoarthritis chondrocyte cell model. CircRNA_Atp9b expression level was elevated in mouse chondrocytes in response to interleukin-1 $\beta$ (Il-1 $\beta$ ) treatment. Suppression of CircRNA_Atp9b expression resulted in decreased matrix metallopeptidase 13 (MMP13), interleukin-6 (IL-6), and cyclooxygenase 2 (COX-2) expression while type II collagen was increased. The regulatory function of circRNA_Atp9b in the progression of osteoarthritis is attributed to the sponging of miR-138-5p in mouse chondrocytes (Zhou et al., 2018a). In a study involving placental tissues to identify circRNA dysregulation in pre-eclampsia by microarray, 143 circRNAs were induced, while 158 were repressed as compared to controls. The top three circRNAs that were significantly upregulated were hsa_circRNA_102682, hsa_circRNA_104820 and hsa_circRNA_100782 (Qian et al., 2016). However the function of these circRNAs in the pathogenesis of pre-eclampsia has not been elucidated to date. Similarly, circ_101222 could be used as pre-eclampsia predictor in combination with the expression levels of endoglin in plasma (Zhang et al., 2016). RNA sequencing involving liver biopsies of chronic hepatitis $B$ virus infection revealed a total of 99 dysregulated circRNAs as compared to control. Computational and regression analyses showed a positive correlation between hsa_circ_0000650 and TGF-beta-2 with a possible interaction between miR-6873$3 \mathrm{p}$ and TGF-beta-2. Taken together these results suggest an involvement of circRNAs in the pathogenesis of hepatitis B virus infection and the progression of liver disease (Zhou T. C. et al., 2018). New evidence implicates circRNAs in aging and cellular senescence. Results of RNA-seq from cortex and hippocampus of young as compared to aged mice showed a significant accumulation of circRNAs in brain tissues (Gruner et al., 2016). The circRNA derived from forkhead box O3 (Foxo3) gene termed circ-Foxo3 is implicated in cellular senescence as overexpression of this circRNA promoted senescence while silencing circ-Foxo3 suppressed cellular senescence. Circ-Foxo3 exerts its effects by binding to cyclin-dependent kinase 2 
(CDK2) and the cell cycle inhibitor p21 (Du et al., 2016, 2017a). Two circRNAs hsa_circ_0005836 and hsa_circ_0009128 were identified using high-throughput sequencing to be downregulated in PBMCs in patients with active pulmonary tuberculosis compared to controls. However, the authors suggest that only hsa_circ_0005836 could be used potentially as a novel tuberculosis biomarker (Zhuang et al., 2017).

\section{CONCLUSION}

The elucidation of the function of circRNAs is an emerging field of science with a tremendous potential after previously being dismissed as RNA artifacts. They are ubiquitously expressed and thousands of members have already been identified. This fact only expands their potential to possibly enhance our knowledge to understand the difference between health and disease. Owing to their structure stability and their presence in exosomes circRNAs may also exert their function in an autocrine, paracrine and possible endocrine fashion. In addition, the fact that circRNAs are widely distributed in the cellular compartment as well as in the extracellular space in various body fluids affords them to become ideal candidates as reliable biomarkers for various human diseases. As in any emerging field caution is advised in interpreting new findings. To date studies are merely descriptive in nature. More in depth analysis about the function and regulation of identified circRNAs is needed to understand their mechanism of action. These RNA molecules hold great promise as biomarkers of disease because of their high resistance to exonuclease activity and might even be more highly expressed than their linear counterparts. Especially their putative role

\section{REFERENCES}

Abu, N., and Jamal, R. (2016). Circular RNAs as promising biomarkers: a minireview. Front. Physiol. 7:355. doi: 10.3389/fphys.2016.00355

Ashwal-Fluss, R., Meyer, M., Pamudurti, N. R., Ivanov, A., Bartok, O., Hanan, M., et al. (2014). circRNA biogenesis competes with pre-mRNA splicing. Mol. Cell 56, 55-66. doi: 10.1016/j.molcel.2014.08.019

Bachmayr-Heyda, A., Reiner, A. T., Auer, K., Sukhbaatar, N., Aust, S., BachleitnerHofmann, T., et al. (2015). Correlation of circular RNA abundance with proliferation-exemplified with colorectal and ovarian cancer, idiopathic lung fibrosis, and normal human tissues. Sci. Rep. 5:8057. doi: 10.1038/srep08057

Bai, N., Peng, E., Qiu, X., Lyu, N., Zhang, Z., Tao, Y., et al. (2018). circFBLIM1 act as a ceRNA to promote hepatocellular cancer progression by sponging miR-346. J. Exp. Clin. Cancer Res. 37:172. doi: 10.1186/s13046-018-0838-8

Bao, X., Zheng, S., Mao, S., Gu, T., Liu, S., Sun, J., et al. (2018). A potential risk factor of essential hypertension in case-control study: circular RNA hsa_circ_0037911. Biochem. Biophys. Res. Commun. 498, 789-794. doi: 10.1016/j.bbrc.2018. 03.059

Bissels, U., Wild, S., Tomiuk, S., Holste, A., Hafner, M., Tuschl, T., et al. (2009). Absolute quantification of microRNAs by using a universal reference. RNA 15, 2375-2384. doi: 10.1261/rna.1754109

Bobrie, A., Colombo, M., Raposo, G., and Thery, C. (2011). Exosome secretion: molecular mechanisms and roles in immune responses. Traffic 12, 1659-1668. doi: $10.1111 / j .1600-0854.2011 .01225 . x$

Brandenburger, T., Salgado Somoza, A., Devaux, Y., and Lorenzen, J. M. (2018). Noncoding RNAs in acute kidney injury. Kidney Int. 94, 870-881. doi: 10.1016/ j.kint.2018.06.033

Cao, Y., Gao, X., Yang, Y., Ye, Z., Wang, E., and Dong, Z. (2018). Changing expression profiles of long non-coding RNAs, mRNAs and circular RNAs as miRNA sponges makes them interesting targets for future investigations. In the future circRNA-based therapies might be introduced. The concept of establishing circRNAs as therapeutics in the future relies on the stability and expression in distinct organs and their specificity for a certain disease. The more specific circRNAs are to a specific organ or disease, the less likely it is to encounter off-target effects, while therapeutically modulating a specific circRNA in a disease setting. As is known for microRNA the likelihood of ensuing significant and undesired off-target effects greatly limits the use of these transcripts as therapeutics. The elucidation of the role of specific circRNAs is the prerequisite for a targeted RNA-based therapy for a specific disease. Tissue/cell specificity might be achieved by coupling ncRNAs to tissue-specific antibodies and/or peptides, thereby reducing off-target effects.

\section{AUTHOR CONTRIBUTIONS}

GH and JL contributed equally to the review manuscript. Both authors approved the final manuscript for submission and publication.

\section{ACKNOWLEDGMENTS}

Support of the Swiss National Science Foundation (SNCF) as well as a junior grant by The Swiss National Centre of Competence in Research (NCCR) Kidney Control of Homeostasis (Kidney.CH), both to JL is acknowledged.

in ethylene glycol-induced kidney calculi rats. BMC Genomics 19:660. doi: 10.1186/s12864-018-5052-8

Capel, B., Swain, A., Nicolis, S., Hacker, A., Walter, M., Koopman, P., et al. (1993). Circular transcripts of the testis-determining gene sry in adult mouse testis. Cell 73, 1019-1030. doi: 10.1016/0092-8674(93)90279-y

Carrara, M., Fuschi, P., Ivan, C., and Martelli, F. (2018). Circular RNAs: Methodological challenges and perspectives in cardiovascular diseases. J. Cell Mol. Med. 22, 5176-5187. doi: 10.1111/jcmm.13789

Carter, M. G., Sharov, A. A., Vanburen, V., Dudekula, D. B., Carmack, C. E., Nelson, C., et al. (2005). Transcript copy number estimation using a mouse whole-genome oligonucleotide microarray. Genome Biol. 6:R61.

Cocquerelle, C., Mascrez, B., Hetuin, D., and Bailleul, B. (1993). Mis-splicing yields circular RNA molecules. FASEB J. 7, 155-160. doi: 10.1096/fasebj.7.1.7678559

Danan, M., Schwartz, S., Edelheit, S., and Sorek, R. (2012). Transcriptome-wide discovery of circular RNAs in Archaea. Nucleic Acids Res. 40, 3131-3142. doi: 10.1093/nar/gkr1009

Du, W. W., Yang, W., Chen, Y., Wu, Z. K., Foster, F. S., Yang, Z., et al. (2017a). Foxo3 circular RNA promotes cardiac senescence by modulating multiple factors associated with stress and senescence responses. Eur. Heart J. 38, 1402-1412. doi: 10.1093/eurheartj/ehw001

Du, W. W., Zhang, C., Yang, W., Yong, T., Awan, F. M., and Yang, B. B. (2017b). Identifying and characterizing circRNA-protein interaction. Theranostics 7, 4183-4191. doi: 10.7150/thno.21299

Du, W. W., Yang, W., Liu, E., Yang, Z., Dhaliwal, P., and Yang, B. B. (2016). Foxo3 circular RNA retards cell cycle progression via forming ternary complexes with p21 and CDK2. Nucleic Acids Res. 44, 2846-2858. doi: 10.1093/nar/gkw027

Duncan, R., and Hershey, J. W. (1983). Identification and quantitation of levels of protein synthesis initiation factors in crude HeLa cell lysates by twodimensional polyacrylamide gel electrophoresis. J. Biol. Chem. 258, 7228-7235. 
Fan, X., Weng, X., Zhao, Y., Chen, W., Gan, T., and Xu, D. (2017). Circular RNAs in cardiovascular disease: an overview. Biomed. Res. Int. 2017:5135781. doi: $10.1155 / 2017 / 5135781$

Fang, Y. (2018). Circular RNAs as novel biomarkers with regulatory potency in human diseases. Future Sci. OA 4:FSO314. doi: 10.4155/fsoa-2018-0036

Fang, Y., Wang, X., Li, W., Han, J., Jin, J., Su, F., et al. (2018). Screening of circular RNAs and validation of circANKRD36 associated with inflammation in patients with type 2 diabetes mellitus. Int. J. Mol. Med. 42, 1865-1874. doi: 10.3892/ijmm.2018.3783

Gruner, H., Cortes-Lopez, M., Cooper, D. A., Bauer, M., and Miura, P. (2016). CircRNA accumulation in the aging mouse brain. Sci. Rep. 6:38907. doi: 10 . 1038/srep38907

Han, D., Li, J., Wang, H., Su, X., Hou, J., Gu, Y., et al. (2017). Circular RNA circMTO1 acts as the sponge of microRNA-9 to suppress hepatocellular carcinoma progression. Hepatology 66, 1151-1164. doi: 10.1002/hep.29270

Hansen, T. B., Jensen, T. I., Clausen, B. H., Bramsen, J. B., Finsen, B., Damgaard, C. K., et al. (2013). Natural RNA circles function as efficient microRNA sponges. Nature 495, 384-388. doi: 10.1038/nature11993

Hansen, T. B., Wiklund, E. D., Bramsen, J. B., Villadsen, S. B., Statham, A. L., Clark, S. J., et al. (2011). miRNA-dependent gene silencing involving Ago2mediated cleavage of a circular antisense RNA. EMBO J. 30, 4414-4422. doi: 10.1038/emboj.2011.359

Hao, L., Rong, W., Bai, L., Cui, H., Zhang, S., Li, Y., et al. (2019). Upregulated circular RNA circ_0007534 indicates an unfavorable prognosis in pancreatic ductal adenocarcinoma and regulates cell proliferation, apoptosis, and invasion by sponging miR-625 and miR-892b. J. Cell Biochem. 120, 3780-3789. doi: $10.1002 /$ jcb. 27658

Haque, S., and Harries, L. W. (2017). Circular RNAs (circRNAs) in health and disease. Genes 8:E353.

Hastie, N. D., and Bishop, J. O. (1976). The expression of three abundance classes of messenger RNA in mouse tissues. Cell 9, 761-774. doi: 10.1016/0092-8674(76) 90139-2

Holdt, L. M., Kohlmaier, A., and Teupser, D. (2018a). Circular RNAs as therapeutic agents and targets. Front. Physiol. 9:1262.

Holdt, L. M., Kohlmaier, A., and Teupser, D. (2018b). Molecular roles and function of circular RNAs in eukaryotic cells. Cell Mol. Life Sci. 75, 1071-1098. doi: 10.1007/s00018-017-2688-5

Holdt, L. M., Stahringer, A., Sass, K., Pichler, G., Kulak, N. A., Wilfert, W., et al. (2016). Circular non-coding RNA ANRIL modulates ribosomal RNA maturation and atherosclerosis in humans. Nat. Commun. 7:12429. doi: $10.1038 /$ ncomms 12429

Huang, S., Yang, B., Chen, B. J., Bliim, N., Ueberham, U., Arendt, T., et al. (2017). The emerging role of circular RNAs in transcriptome regulation. Genomics 109, 401-407. doi: 10.1016/j.ygeno.2017.06.005

Jeck, W. R., and Sharpless, N. E. (2014). Detecting and characterizing circular RNAs. Nat. Biotechnol. 32, 453-461. doi: 10.1038/nbt.2890

Jeck, W. R., Sorrentino, J. A., Wang, K., Slevin, M. K., Burd, C. E., Liu, J., et al. (2013). Circular RNAs are abundant, conserved, and associated with ALU repeats. RNA 19, 141-157. doi: 10.1261/rna.035667.112

Kolling, M., Seeger, H., Haddad, G., Kistler, A., Nowak, A., Faulhaber-Walter, R., et al. (2018). The Circular RNA ciRs-126 predicts survival in critically patients with acute kidney injury Ill. Kidney Int. Rep. 3, 1144-1152. doi: 10.1016/j.ekir. 2018.05.012

Kristensen, L. S., Hansen, T. B., Veno, M. T., and Kjems, J. (2018). Circular RNAs in cancer: opportunities and challenges in the field. Oncogene 37, 555-565. doi: 10.1038/onc.2017.361

Lan, P. H., Liu, Z. H., Pei, Y. J., Wu, Z. G., Yu, Y., Yang, Y. F., et al. (2016). Landscape of RNAs in human lumbar disc degeneration. Oncotarget 7 , 63166-63176. doi: 10.18632/oncotarget.11334

Li, G., Huang, M., Cai, Y., Yang, Y., Sun, X., and Ke, Y. (2019). Circ-U2AF1 promotes human glioma via derepressing neuro-oncological ventral antigen 2 by sponging hsa-miR-7-5p. J. Cell Physiol. 234, 9144-9155. doi: 10.1002/jcp. 27591

Li, L., Guo, L., Yin, G., Yu, G., Zhao, Y., and Pan, Y. (2018). Upregulation of circular RNA circ_0001721 predicts unfavorable prognosis in osteosarcoma and facilitates cell progression via sponging miR-569 and miR-599. Biomed. Pharmacother. 109, 226-232. doi: 10.1016/j.biopha.2018.10.072
Li, P., Yang, X., Yuan, W., Yang, C., Zhang, X., Han, J., et al. (2018). CircRNACdrlas exerts anti-oncogenic functions in bladder cancer by sponging microRNA-135a. Cell Physiol. Biochem. 46, 1606-1616. doi: 10.1159/000489208

Li, Y., Hu, J., Li, L., Cai, S., Zhang, H., Zhu, X., et al. (2018). Upregulated circular RNA circ_0016760 indicates unfavorable prognosis in NSCLC and promotes cell progression through miR-1287/Gagel axis. Biochem. Biophys. Res. Commun. 503, 2089-2094. doi: 10.1016/j.bbrc.2018.07.164

Li, Y., Zheng, Q., Bao, C., Li, S., Guo, W., Zhao, J., et al. (2015). Circular RNA is enriched and stable in exosomes: a promising biomarker for cancer diagnosis. Cell Res. 25, 981-984. doi: 10.1038/cr.2015.82

Li, Z., Huang, C., Bao, C., Chen, L., Lin, M., Wang, X., et al. (2015). Exon-intron circular RNAs regulate transcription in the nucleus. Nat. Struct. Mol. Biol. 22, 256-264. doi: 10.1038/nsmb.2959

Lorenzen, J. M., and Thum, T. (2016). Long noncoding RNAs in kidney and cardiovascular diseases. Nat. Rev. Nephrol. 12, 360-373. doi: 10.1038/nrneph. 2016.51

Lu, Y., Qin, B., Hu, H., Zhang, J., Wang, Y., Wang, Q., et al. (2016). Integrative microRNA-gene expression network analysis in genetic hypercalciuric stoneforming rat kidney. PeerJ 4:e1884. doi: 10.7717/peerj.1884

Luan, J., Jiao, C., Kong, W., Fu, J., Qu, W., Chen, Y., et al. (2018). circHLA-C plays an important role in lupus nephritis by sponging miR-150. Mol. Ther. Nucleic Acids 10, 245-253. doi: 10.1016/j.omtn.2017.12.006

Memczak, S., Jens, M., Elefsinioti, A., Torti, F., Krueger, J., Rybak, A., et al. (2013). Circular RNAs are a large class of animal RNAs with regulatory potency. Nature 495, 333-338. doi: 10.1038/nature11928

Memczak, S., Papavasileiou, P., Peters, O., and Rajewsky, N. (2015). Identification and characterization of circular RNAs As a new class of putative biomarkers in human blood. PLoS One 10:e0141214. doi: 10.1371/journal.pone.0141214

Mortazavi, A., Williams, B. A., Mccue, K., Schaeffer, L., and Wold, B. (2008). Mapping and quantifying mammalian transcriptomes by RNA-Seq. Nat. Methods 5, 621-628. doi: 10.1038/nmeth.1226

Nicolet, B. P., Engels, S., Aglialoro, F., Van Den Akker, E., Von Lindern, M., and Wolkers, M. C. (2018). Circular RNA expression in human hematopoietic cells is widespread and cell-type specific. Nucleic Acids Res. 46, 8168-8180. doi: 10.1093/nar/gky721

Palazzo, A. F., and Lee, E. S. (2015). Non-coding RNA: what is functional and what is junk? Front. Genet. 6:2. doi: 10.3389/fgene.2015.00002

Peng, Y. T., Song, X. J., Zheng, Y., Cheng, H. Y., and Lai, W. (2018). circCOL3A1859267 regulates type I collagen expression by sponging miR-29c in human dermal fibroblasts. Eur. J. Dermatol. 28, 613-620. doi: 10.1684/ejd.2018.3397

Properzi, F., Logozzi, M., and Fais, S. (2013). Exosomes: the future of biomarkers in medicine. Biomark Med. 7, 769-778. doi: 10.2217/bmm.13.63

Qian, Y., Lu, Y., Rui, C., Qian, Y., Cai, M., and Jia, R. (2016). Potential significance of circular RNA in human placental tissue for patients with preeclampsia. Cell Physiol. Biochem. 39, 1380-1390. doi: 10.1159/000447842

Raposo, G., and Stoorvogel, W. (2013). Extracellular vesicles: exosomes, microvesicles, and friends. J. Cell Biol. 200, 373-383. doi: 10.1083/jcb. 201211138

Ruskin, B., and Green, M. R. (1990). RNA lariat debranching enzyme as tool for analyzing RNA structure. Methods Enzymol. 181, 180-188. doi: 10.1016/00766879(90)81120-j

Salzman, J. (2016). Circular RNA expression: its potential regulation and function. Trends Genet. 32, 309-316. doi: 10.1016/j.tig.2016.03.002

Salzman, J., Gawad, C., Wang, P. L., Lacayo, N., and Brown, P. O. (2012). Circular RNAs are the predominant transcript isoform from hundreds of human genes in diverse cell types. PLoS One 7:e30733. doi: 10.1371/journal.pone.0030733

Stępień, E., Costa, M. C., Kurc, S., Drozdz, A., Cortez-Dias, N., and Enguita, F. J. (2018). The circulating non-coding RNA landscape for biomarker research: lessons and prospects from cardiovascular diseases. Acta Pharmacol. Sin. 39, 1085-1099. doi: 10.1038/aps.2018.35

Sun, M., Zhao, W., Chen, Z., Li, M., Li, S., Wu, B., et al. (2019). Circ_0058063 regulates CDK6 to promote bladder cancer progression by sponging miR-1455p. J. Cell Physiol. 234, 4812-4824. doi: 10.1002/jcp.27280

Suzuki, H., Zuo, Y., Wang, J., Zhang, M. Q., Malhotra, A., and Mayeda, A. (2006). Characterization of RNase R-digested cellular RNA source that consists of lariat and circular RNAs from pre-mRNA splicing. Nucleic Acids Res. 34:e63. doi: $10.1093 / \mathrm{nar} / \mathrm{gkl151}$ 
Tabak, H. F., Van Der Horst, G., Smit, J., Winter, A. J., Mul, Y., and Groot Koerkamp, M. J. (1988). Discrimination between RNA circles, interlocked RNA circles and lariats using two-dimensional polyacrylamide gel electrophoresis. Nucleic Acids Res. 16, 6597-6605. doi: 10.1093/nar/16.14.6597

Tang, C. M., Zhang, M., Huang, L., Hu, Z. Q., Zhu, J. N., Xiao, Z., et al. (2017). CircRNA_000203 enhances the expression of fibrosis-associated genes by derepressing targets of miR-26b-5p, Colla2 and CTGF, in cardiac fibroblasts. Sci. Rep. 7:40342. doi: 10.1038/srep40342

Van Der Pol, E., Boing, A. N., Harrison, P., Sturk, A., and Nieuwland, R. (2012). Classification, functions, and clinical relevance of extracellular vesicles. Pharmacol. Rev. 64, 676-705. doi: 10.1124/pr.112.005983

Vicens, Q., and Westhof, E. (2014). Biogenesis of circular RNAs. Cell 159, 13-14. doi: 10.1016/j.cell.2014.09.005

Vo, J. N., Cieslik, M., Zhang, Y., Shukla, S., Xiao, L., Zhang, Y., et al. (2019). The landscape of circular RNA in cancer. Cell 176:e13. doi: 10.1016/j.cell.2018. 12.021

Waldron, C., and Lacroute, F. (1975). Effect of growth rate on the amounts of ribosomal and transfer ribonucleic acids in yeast. J. Bacteriol. 122, 855-865.

Wang, K., Gan, T. Y., Li, N., Liu, C. Y., Zhou, L. Y., Gao, J. N., et al. (2017). Circular RNA mediates cardiomyocyte death via miRNA-dependent upregulation of Mtp18 expression. Cell Death Differ. 24, 1111-1120. doi: 10.1038/cdd.2017.61

Wang, X., Dai, Y., Zhang, W., Sun, D., and Zhang, X. (2017). Microarray based circRNA expression profiles in uremic plasma and PMBCs due to chronic glomerulonephritis. Arch. Biol. Sci. 69, 523-534. doi: 10.2298/abs160520128w

Althesha, M. A., Ni, T., Khan, A., Liu, K., and Zheng, X. (2018). Circular RNA in cardiovascular disease. J. Cell. Physiol. 234, 5588-5600. doi: 10.1002/jcp.27384

Wang, P. L., Bao, Y., Yee, M. C., Barrett, S. P., Hogan, G. J., Olsen, M. N., et al. (2014). Circular RNA is expressed across the eukaryotic tree of life. PLoS One 9:e90859. doi: 10.1371/journal.pone.0090859

Wang, S., Li, Q., Wang, Y., Li, X., Wang, R., Kang, Y., et al. (2018). Upregulation of circ-UBAP2 predicts poor prognosis and promotes triple-negative breast cancer progression through the miR-661/MTA1 pathway. Biochem. Biophys. Res. Commun. 505, 996-1002. doi: 10.1016/j.bbrc.2018.10.026

Wang, Y., Li, M., Wang, Y., Liu, J., Zhang, M., Fang, X., et al. (2019). A Zfp609 circular RNA regulates myoblast differentiation by sponging miR-194-5p. Int. J. Biol. Macromol. 121, 1308-1313. doi: 10.1016/j.ijbiomac.2018.09.039

Wilusz, J. E. (2018). A 360 degrees view of circular RNAs: from biogenesis to functions. Wiley Interdiscip. Rev. RNA 9:e1478. doi: 10.1002/wrna.1478

Wolf, S. F., and Schlessinger, D. (1977). Nuclear metabolism of ribosomal RNA in growing, methionine-limited, and ethionine-treated HeLa cells. Biochemistry 16, 2783-2791. doi: 10.1021/bi00631a031

Xie, B., Zhao, Z., Liu, Q., Wang, X., Ma, Z., and Li, H. (2018). CircRNA has_circ_0078710 acts as the sponge of microrna-31 involved in hepatocellular carcinoma progression. Gene 683, 253-261. doi: 10.1016/j.gene.2018.10.043

Xu, J. Y., Chang, N. B., Rong, Z. H., Li, T., Xiao, L., Yao, Q. P., et al. (2018). CircDiaph3 regulates rat vascular smooth muscle cell differentiation, proliferation, and migration. FASEB J. 33, 2659-2668. doi: 10.1096/fj. 201800243RRR

Xu, T., Wu, J., Han, P., Zhao, Z., and Song, X. (2017). Circular Rna expression profiles and features in human tissues: a study using RNA-seq data. BMC Genomics 18:680. doi: 10.1186/s12864-017-4029-3

Yang, C., Wu, S., Wu, X., Zhou, X., Jin, S., and Jiang, H. (2019). Silencing circular RNA UVRAG inhibits bladder cancer growth and metastasis by targeting the microRNA-223/fibroblast growth factor receptor 2 axis. Cancer Sci. 110, 99-106. doi: 10.1111/cas.13857

Yang, C., Yuan, W., Yang, X., Li, P., Wang, J., Han, J., et al. (2018). Circular RNA circ-ITCH inhibits bladder cancer progression by sponging miR-17/miR-224 and regulating p21. PTEN Exp. Mol. Cancer 17:19. doi: 10.1186/s12943-0180771-7

Yang, Y., Gao, X., Zhang, M., Yan, S., Sun, C., Xiao, F., et al. (2018b). Novel role of FBXW7 circular RNA in repressing glioma tumorigenesis. J. Natl. Cancer Inst. 110, 304-315. doi: 10.1093/jnci/djx166
Yang, Y., Fan, X., Mao, M., Song, X., Wu, P., Zhang, Y., et al. (2017). Extensive translation of circular RNAs driven by N(6)-methyladenosine. Cell Res. 27, 626-641. doi: 10.1038/cr.2017.31

Yuan, Y., Liu, W., Zhang, Y., Zhang, Y., and Sun, S. (2018). CircRNA circ_0026344 as a prognostic biomarker suppresses colorectal cancer progression via microRNA-21 and microRNA-31. Biochem. Biophys. Res. Commun. 503, 870-875. doi: 10.1016/j.bbrc.2018.06.089

Zhang, S. J., Chen, X., Li, C. P., Li, X. M., Liu, C., Liu, B. H., et al. (2017). Identification and characterization of circular RNAs as a new class of putative biomarkers in diabetes retinopathy. Invest. Ophthalmol. Vis. Sci. 58, 6500-6509. doi: 10.1167/iovs.17-22698

Zhang, Y., Zhang, X. O., Chen, T., Xiang, J. F., Yin, Q. F., Xing, Y. H., et al. (2013). Circular intronic long noncoding RNAs. Mol. Cell 51, 792-806. doi: 10.1016/j.molcel.2013.08.017

Zhang, Y. G., Yang, H. L., Long, Y., and Li, W. L. (2016). Circular RNA in blood corpuscles combined with plasma protein factor for early prediction of pre-eclampsia. BJOG 123, 2113-2118. doi: 10.1111/1471-0528.13897

Zheng, Q., Bao, C., Guo, W., Li, S., Chen, J., Chen, B., et al. (2016). Circular RNA profiling reveals an abundant circHIPK3 that regulates cell growth by sponging multiple miRNAs. Nat. Commun. 7:11215. doi: 10.1038/ncomms11215

Zhong, L., Wang, Y., Cheng, Y., Wang, W., Lu, B., Zhu, L., et al. (2018). Circular RNA circC3P1 suppresses hepatocellular carcinoma growth and metastasis through miR-4641/PCK1 pathway. Biochem. Biophys. Res. Commun. 499, 1044-1049. doi: 10.1016/j.bbrc.2018.03.221

Zhou, B., and Yu, J. W. (2017). A novel identified circular RNA, circRNA_010567, promotes myocardial fibrosis via suppressing miR-141 by targeting TGFbeta1. Biochem. Biophys. Res. Commun. 487, 769-775. doi: 10.1016/j.bbrc.2017. 04.044

Zhou, L. Y., Zhai, M., Huang, Y., Xu, S., An, T., Wang, Y. H., et al. (2018). The circular RNA ACR attenuates myocardial ischemia/reperfusion injury by suppressing autophagy via modulation of the Pink1/ FAM65B pathway. Cell Death Differ. doi: 10.1038/s41418-018-0206-4 [Epub ahead of print].

Zhou, M. Y., Yang, J. M., and Xiong, X. D. (2018). The emerging landscape of circular RNA in cardiovascular diseases. J. Mol. Cell. Cardiol. 122, 134-139. doi: 10.1016/j.yjmcc.2018.08.012

Zhou, T. C., Li, X., Chen, L. J., Fan, J. H., Lai, X., Tang, Y., et al. (2018). Differential expression profile of hepatic circular RNAs in chronic hepatitis B. J. Viral. Hepat. 25, 1341-1351. doi: 10.1111/jvh.12944

Zhou, Z. B., Du, D., Huang, G. X., Chen, A., and Zhu, L. (2018a). Circular RNA Atp9b, a competing endogenous RNA, regulates the progression of osteoarthritis by targeting miR-138-5p. Gene 646, 203-209. doi: 10.1016/j.gene. 2017.12.064

Zhou, Z. B., Niu, Y. L., Huang, G. X., Lu, J. J., Chen, A., and Zhu, L. (2018b). Silencing of circRNA.2837 plays a protective role in sciatic nerve injury by sponging the miR-34 family via regulating neuronal autophagy. Mol. Ther. Nucleic Acids 12, 718-729. doi: 10.1016/j.omtn.2018.07.011

Zhuang, Z. G., Zhang, J. A., Luo, H. L., Liu, G. B., Lu, Y. B., Ge, N. H., et al. (2017). The circular RNA of peripheral blood mononuclear cells: Hsa_circ_0005836 as a new diagnostic biomarker and therapeutic target of active pulmonary tuberculosis. Mol. Immunol. 90, 264-272. doi: 10.1016/j.molimm.2017. 08.008

Conflict of Interest Statement: The authors declare that the research was conducted in the absence of any commercial or financial relationships that could be construed as a potential conflict of interest.

Copyright (c) 2019 Haddad and Lorenzen. This is an open-access article distributed under the terms of the Creative Commons Attribution License (CC BY). The use, distribution or reproduction in other forums is permitted, provided the original author(s) and the copyright owner(s) are credited and that the original publication in this journal is cited, in accordance with accepted academic practice. No use, distribution or reproduction is permitted which does not comply with these terms. 